\title{
Do Brasil ao Québec, alguns elementos para reflexão: Os Agentes Comunitários de Saúde conectam famílias e os profissionais de saúde. ${ }^{1}$
}

\author{
From Brazil to Quebec, some issues for reflection: \\ Community health agents connect families to \\ health professionals.
}

\section{De Brasil à Québec, algunos elementos para la reflexión: los Agentes Comunitarios de Salud vinculan a las familias con los profesionales de salud.}

\author{
Lise RENAUD ${ }^{2}$ \\ Margareth Santos ZANCHETTA ${ }^{3}$ \\ Jacques RHÉAUME ${ }^{4}$ \\ Roger CÔTÉ 5
}

RESUMO: O sistema único de saúde brasileiro comporta 27.000 equipes de saúde da família com uma rede de cerca de 300.000 Agentes Comunitários de Saúde cuja responsabilidade é a de conectar as famílias que são visitadas mensalmente com os profissionais de saúde da unidade de saúde da família. Desde 2003, o Québec realiza uma reforma no setor da saúde e serviços sociais com ênfase na integração de cuidados e serviços (públicos, associativos e privados) e sua inscrição nas redes locais segundo uma perspectiva de responsabilidade populacional. Os comentários apresentados tentam destacar alguns elementos de semelhança ou diferença entre o programa das Unidades de 1 Artigo originalmente publicado como: Renaud, L., Zanchetta, M., Rhéaume, J., \& Côté, R. (2011). Du Brésil au Québec, quelques éléments de réflexion: les agents communautaires de santé brésiliens font le pont entre les familles et les professionnels de la santé. (Commentaire). Canadian Journal of Public Health / Revue canadienne de santé publique, 102(1), 61-63. Tradução autorizada pela Revista. Correspondência: Dra. Lise Renaud, UQAM, case postale 8888, Centre Ville Montréal (Québec) H3C 3P8, email : renaud.lise@uqam.ca.

2 Doutor (a) Professor (a) Titular Departamento de comunicação social e política, Faculdade de Comunicação, Universite du Québec à Montréal (UQAM), Montréal, QC, Canadá. Pesquisador (a) Centro de saúde e de serviços sociais de la Montagne, Montréal, QC, Canadá.

3 Doutora, Professora Adjunta, Escola de Enfermagem Daphne Cockwell, Faculdade de Serviços Comunitários, Ryerson University, Toronto, ON, Canadá. Pesquisador (a) Centro de saúde e de serviços sociais de la Montagne, Montréal, QC, Canadá.

4 Doutor (a) Professor (a) Titular Departamento de comunicação social e política, Faculdade de Comunicação, Universite du Québec à Montréal (UQAM), Montréal, QC, Canadá. Pesquisador (a) Centro de saúde e de serviços sociais de la Montagne, Montréal, QC, Canadá.

4 Bacharel, Agente de planejamento, Centro de Pesquisa e de Educação, Centro de saúde e de serviços sociais de la Montagne, Montréal, QC, Canadá. Pesquisador (a) Centro de saúde e de serviços sociais de la Montagne, Montréal, QC, Canadá. 
saúde da família implantado no Brasil e aquele das Clínicas da rede integrada ou dos Grupos de medicina de família do Québec. Nos dois casos, existe a preocupação de oferecer serviços de maior aproximação com a população de um dado território, de uma maior acessibilidade e adequação de serviços. Além disto, o Brasil através dos Agentes Comunitários de Saúde, permite uma linha social que assegura o acompanhamento real de todos os membros da família pelos serviços de saúde. Palavras chave: Sistema de Saúde Brasileiro. Agentes Comunitários de Saúde. Profissionais da Saúde.

ABSTRACT: The Brazilian unified health system consists of 27,000 family health teams with a network of approximately 300, 000 community health agents whose mandate is to link the families they visit every month with health professionals at the family health unit. Since 2003, Quebec has been implementing its health and social services reforms by emphasizing the integration of care and services (public, associative and private) in local networks which have a formal population health responsibility. This commentary aims to underline some similarities and differences between the program of the Brazilian 'units of family health' and that of integrated network clinics or groups of family medicine in Quebec. In both cases, a major concern is to organize primary care services in as close proximity as possible to the population of a given territory, and to improve accessibility and adequacy of services. In addition, through these community health agents, Brazil enables the creation of a social security network that assures an effective follow-up of all members of the publication with regard to health services. Keywords: Brazilian Health Care System. Community Health Agents. Health professionals

RESUMEN: El sistema único de salud brasileño posee 27.000 equipos de salud de la familia y una red de aproximadamente 300.000 Agentes Comunitarios de Salud. La función de los Agentes Comunitarios es vincular a las familias, que visitan todos los meses, con profesionales de los equipos de salud de la familia. Desde 2003, Québec ha implementando reformas de los servicios sociales y de salud, haciendo hincapié en la integración de la atención y los servicios (públicos, asociativos y privados) en redes locales que tienen la responsabilidad formal de salud de la población. Este trabajo tiene como objetivo destacar algunas similitudes y diferencias entre el programa de 'unidades de salud de la familia' de Brasil y el de las clínicas integradas en red o grupos de medicina familiar en Québec. En ambos casos, existen las mismas preocupaciones; la de ofrecer servicios más próximos a una población en un territorio determinado, la de una mayor accesibilidad y adecuación de los servicios hacia la comunidad. Además de esto, Brasil, a través de los Agentes Comunitarios de Salud, permite una línea social que asegura el acompañamiento real de todos los miembros de las familias por parte de los servicios de salud. Palabras claves: Sistema Único de Salud Brasileño. Agentes Comunitarios de Salud. Profesionales de la Salud.

\section{COMENTÁRIO}

Em 1988, o Brasil criou o Sistema Único de Saúde (SUS) que na época se inspirava também 
no sistema de saúde universal canadense, apesar da diferença maior de permitir a participação complementar do sistema de saúde privado. Hoje, o Brasil possui uma rede de 27.000 equipes de saúde da família. O medico de família é apoiado por outros profissionais: enfermeiros, nutricionistas, assistentes sociais, entre outros. A ênfase é dada não apenas no médico de família, mas sob as equipes que integram os Agentes Comunitários de Saúde (ACS) (n=300.000) cuja responsabilidade é o de estabelecer uma ponte entre as famílias visitadas mensalmente e os profissionais da unidade da saúde da família. ${ }^{1-9}$ Em decorrência da implantação deste sistema, a mortalidade infantil reduziu a tal ponto, que em 2008 a Organização Mundial da Saúde ${ }^{10}$ sugeriu aos demais países a se inspirarem no modelo do programa brasileiro de saúde da família.

Por ocasião de um projeto sobre determinantes sociais da saúde financiado pelos Institutos Canadenses de Pesquisa em Saúde, alguns elementos das funções dos ACS nos intrigaram em relação ao sistema de saúde do Québec. No Québec, a última reforma de saúde e serviços sociais ${ }^{11}$ visa à integração de serviços (públicos, associativos e privados) e de inseri-los na rede locais segundo uma perspectiva de responsabilidade populacional compartilhada entre os diferentes provedores de serviços com o objetivo de aumentar o acesso e qualidade dos mesmos. Esta reforma oferece pontos de similaridade com o SUS implantado no Brasil.

Mais especificamente, é interessante de examinar o programa implantado nas unidades de saúde da família (USF) e as redes integradas de clinicas ou grupos de medicina familiar no Québec, que devem trabalhar coadunamente com as organizações comunitárias e cujo, objetivo é o de estarem mais próximos das famílias. Nos dois casos, existe a preocupação por serviços de maior proximidade com a população de um dado território, de maior acesso e adequação de servicos ${ }^{7,12}$, ${ }^{13}$. Faz-se necessário ressaltar que os ACS estabelecem um nível de aproximação com os membros da comunidade que é quase inexistente no Québec, exceto nos casos de famílias consideradas como sendo socialmente vulneráveis e que possuem crianças de 0 a 5 anos (programas de serviços integrados de puericultura) e idosos ou portadores de deficiências associadas a uma doença que os beneficie de cuidados domiciliares a curto ou longo termo ${ }^{14}$. Mesmo que estes programas do Québec permitam uma certa aproximação com o sistema brasileiro, os ACS orientam sua ações para a família e não a pessoa vulnerável. Além disto, cada ACS oriundo da comunidade a qual, ele assiste e visita mensalmente. Em consequência, as populações vulneráveis são bem seguidas e acompanhadas pelos serviços de saúde no momento oportuno. Existe assim, uma malha social estendida e mantida que reflete uma vontade de disponibilizar os serviços de saúde a todos, e, de assegurar-se que toda pessoa seja suscetível a ser assistida. Observemos dos aspectos: a proximidade cultural já que o ACS pode ser oriundo da mesma comunidade e a proximidade física, que o ACS visita a família mensalmente.

No Québec, mesmo nas diversas experiências dos grupos de medicina de família ou de redes integradas de clínicas, em alguns casos, as clínicas ditas comunitárias localizadas nos bairros, tratam-se sempre de profissionais de saúde que na maioria dos casos, não reside no território e 
não tem necessariamente nenhuma afiliação a comunidade local com a qual, ele trabalha. Alguns profissionais possuem uma rede informal de referência a certas organizações comunitárias que permite um maior acesso aos serviços, mas que se restringem a instituições existentes tais como a escola, e mais raramente, aos pais ou os vizinhos. Sobretudo, não existe uma missão específica e as referências permanecem aleatórias na maioria das vezes. As organizações comunitárias nos bairros são geralmente próximas da população, mas elas focalizam os diversos membros da comunidade segundo sua missão. Tais organizações não são também responsáveis por um território em sua totalidade. Algumas exceções merecem atenção maior: o trabalho realizado pelos trabalhadores de rua com jovens e outras pessoas oriundas de ambientes vulneráveis, assim como, os organizadores comunitários atuando nas habitações populares. Ainda assim, resta uma zona indefinida mais ou menos importante da população pouco visitada ou alvo de investimento de saúde, e que, por conseguinte, utiliza os serviços de saúde de modo tardio ou mínimo.

Poderíamos esperar neste contexto responder as exigências de responsabilidade populacional dos centros de saúde e de serviços sociais (CSSS) e a implantação de uma rede comparável aquela dos ACS? Não é no mesmo sentido, por exemplo, a ação comunitária em um bairro para poder desenvolver a informação necessária sobre serviços e promover seu acesso para todos os residentes? Algumas condições específicas deveriam ser observadas em tal projeto social.

Sem duvida é possível mobilizar voluntários de um bairro ou de áreas especificas para exercer um papel comparável ao do $\mathrm{ACS}^{15}$. Todavia, as especificidades do sistema de saúde e de serviços sociais do Québec tornam esta iniciativa delicada sob três aspectos:

- É arriscado realizar um levantamento regular e sistemático das necessidades e proceder ao seguimento de famílias por tais "agentes", sem que sejamos acusados de intromissão ou de invasão da vida privada. Para tal, seria necessária uma colaboração voluntária e permitida.

- A implantação de uma rede local de ACS necessitaria de uma formação educacional adequado, com financiamento suficiente e que correspondesse a normas trabalhistas e de administração que não afastasse os ACS de seus locais de afiliação / origem, assegurando-se a qualidade da intervenção profissional. A referência a redes de pares ou de ajuda mútua é um bom ponto de referencia para alcançar tal equilíbrio em tal intervenção. Em todo caso, um orçamento importante deveria ser previsto e, a necessidade inquestionável de mobilizarem-se outros atores sociais, assim como as municipalidades.

- A adaptação ao Québec das responsabilidades do ACS seria de ligar diretamente as preocupações sociais (condições de vida, habitação, trabalho, isolamento social) aos serviços de saúde ou de higiene.

Talvez seria uma vantagem possível no contexto onde a posição dos ACS seja menos submissa, 
nos bairros, às pressões sociais que limitem as intervenções as únicas prioridades de higiene ou de serviços primários de saúde, mas isto não é evidente. Por exemplo, a afiliação dos ACS a um meio especifico dificulta a distancia e a autonomia, necessárias nos casos de denúncias de situações inaceitáveis, sem comprometer os laços de confiança e a abertura com as pessoas locais. Além disto, uma ligação muito direta com os grupos de medicina de família ou as redes de clinicas integradas poderiam levar ao risco de afastar os ACS de seus modos de ação mais causadas no meio de afiliação. O desenvolvimento de organizações comunitárias autônomas em parceiras, em relação com as clínicas e outros serviços sociais poderia ser uma alternativa mais apropriada seguindo as dinâmicas dos diversos bairros urbanos.

Permanece ainda a diferença essencial entre o sistema atual de saúde do Québec e o desenvolvimento da saúde comunitária segundo o modelo brasileiro. Neste último, a ênfase não é colocada sob o médico de família, mas sob as equipes das quais, integram os ACS com o objetivo de estar o mais perto possível da população e de estar em contato com as pessoas vulneráveis. Como citado em um artigo do jornal Le Devoir ${ }^{17}$ : «Uma mudança audaciosa de paradigma que permitiu de orientar a saúde não mais em torno do hospital sacro-santo, mas à sua base, diretamente sob a primeira linha, seja relacionado ao que chamamos de cuidados primários». Assim, esta diferença entre os dois sistemas de saúde brasileiro e quebequense permanece na abordagem proativa que o Brasil adotou a fim de contatar sua população. No Brasil, os serviços de saúde decidiram de buscar a população em seu ambiente para falar-lhe de saúde, de prevenção, para conduzi-la aos serviços de saúde e, se ela resiste a se mobilizar, os ACS tentarão o que for possível para responder as necessidades das famílias. Existe uma vontade bem firme que não existe no Québec. A partir do momento em que os CSSS terminem a implantação de todos seus projetos clínicos tal que exigido pela reforma, será apenas uma pequena parcela da populacional do território que será coberta por tais programas e toda a energia de seu pessoal será consagrada a tais projetos.

Nosso projeto de disseminação de conhecimentos sobre determinantes sociais de saúde no Brasil nos fez ver principalmente, a implantação de serviços de saúde e de serviços sociais atuais que não visam alcançar o conjunto da população e de soluções a serem propostas. Talvez, devem-se desenvolver mais um novo programa concebido segundo uma abordagem baseada na colaboração com as comunidades, principalmente aquelas de meios vulneráveis e aquelas que sofrem com problemas crônicos de saúde, para sustentá-las e favorecer a implantação de condições favoráveis para melhorar sua saúde, como nos incita a fazer os dispositivos legais atuais.

\section{REFERÊNCIAS BIBLIOGRÁFICAS}

1. Aquino R, Medina MG, Villasbôas AL, Baqueiro CCD, Nunes CA, Caputo MC, et al. Treinamento introdutório das equipes de saúde da família (Manual Técnico). 2001.

2. Ministério da Saúde, Secretaria de Assistência à Saúde, Coordenação de Saúde da Comunidade. Saúde da Família: uma estratégia para a reorganização do modelo assistencial (2a 
ed.). Brasília, Ministério da Saúde, 1998.

3. Ministério da Saúde. Manual para Organização da Atenção Básica. Brasília, Ministério da Saúde, 1999.

4. Ministério da Saúde. Saúde da Família no Brasil: linhas estratégicas para o quadriênio 1999/2002 (resumo executivo). Brasília, Ministério da Saúde, 1999.

5. Ministério da Saúde, Secretaria de Assistência à Saúde, Coordenação de Saúde da Comunidade. Avaliação da implantação e funcionamento do Programa de Saúde da Família. Brasília, Ministério da Saúde, 2000.

6. Conselho Nacional de Secretários de Saúde (CONASS). Legislação do SUS. Brasília, 2003.

7. Hartz ZMA, Contandriopoulos AP. Integralidade da atenção e integração de serviços de saúde; desafios para avaliar a implantação de um « sistema sem muros » (mimeo). 2004.

8. Medina MG, Nascimento EL, Lima MS, Impallari C, Rodrigues RM, Aquino R. Sistema de Informação da Atenção Básica: Indicadores 1999 (Caderno Técnico). Brasília, Ministério da Saúde, 2000.

9. Medina MG, Pereira RAG, Hartz ZMA, Silva LMZ. O Programa de Saúde da Família: evolução de sua implantação no Brasil. A produção sobre saúde da família, Trabalhos apresentados no VII, Congresso Brasileiro de Saúde Coletiva (ABRASCO), Ministério da Saúde, 2003.

10. Organisation mondiale de la santé (OMS). Les soins de santé primaires - maintenant plus que jamais. Organisation mondiale de la santé, hors série, 2008. Internet : http://www.who.int/ whr/2008/summary/fr/index.html (consultado em 24 février 2010).

11. Ministère de la Santé et des Services sociaux (MSSS). Projet clinique : Cadre de référence pour les réseaux locaux de services de santé et de services sociaux : Document principal. Québec, Ministère de la Santé et des Services sociaux, 2004.

12. Contandriopoulos AP. Les enjeux éthiques associés à la transformation des systèmes de soins. Ethique publique 2003;5(1):42-57.

13. Paim JS. Saúde da família: espaço de reflexão e de práticas contra-hegemônicas? Em: Paim JS (éd.), Saúde: Política e reforma sanitária. Salvador, Instituto de Saúde Coletiva, 2002.

14. Ministère de la Santé et des Services sociaux. Les services integrés en périnatalité et pour la petite enfance à l'intention des familles vivant en contexte de vulnérabilité : Cadre de référence. Québec, 2004.

15. Lancman S, Uchida S, Sznelwar LI, Jardim TA. Agente comunitário de saúde: um trabalhador na berlinda. Estudo em psicodinâmica do trabalho. Travailler: revue internationale de psychopathologie et psychodynamique du travail 2007;17:71-124.

16. Côté R, Renaud L, Rhéaume J, Zanchetta M. Retour de mission: visite au Brésil pour // Tempus, actas de saúde colet, Brasília, 8(3), 11-17, set, 2014.

ISSN 1982-8829 
disséminer de l'information sur les déterminants sociaux de la santé et les vulnérabilités sociales. Cahiers METISS 2010; 5(1): 47-60.

17. Rioux Soucy LM. Quand « la santé pour tous » devient réalité. Le Devoir 6 février 2010; Sect. Santé.

Artigo apresentado em 04/03/14

Artigo aprovado em 08/08/14

Artigo publicado no sistema em 12/09/14 